\title{
Laser-plasma lens for laser-wakefield accelerators
}

\author{
R. Lehe, ${ }^{*}$ C. Thaury, ${ }^{\dagger}$ E. Guillaume, A. Lifschitz, and V. Malka \\ Laboratoire d'Optique Appliquée, ENSTA-CNRS UMR7639-École Polytechnique, \\ Chemin de la Hunière, 91761 Palaiseau, France
}

(Received 26 June 2014; published 12 December 2014)

\begin{abstract}
Thanks to their compactness and unique properties, laser-wakefield accelerators are currently considered for several innovative applications. However, many of these applications - and especially those that require beam transport_-are hindered by the large divergence of laser-accelerated beams. Here we propose a collimating concept that relies on the strong radial electric field of the laser-wakefield to reduce this divergence. This concept utilizes an additional gas jet, placed after the laser-wakefield accelerator. When the laser pulse propagates through this additional gas jet, it drives a wakefield which can refocus the trailing electron bunch. Particle-in-cell simulations demonstrate that this approach can reduce the divergence by at least a factor of 3 for realistic electron bunches.
\end{abstract}

DOI: 10.1103/PhysRevSTAB.17.121301

PACS numbers: 52.38.Kd, 52.65.Rr, 41.75.Jv

\section{INTRODUCTION}

Laser-wakefield accelerators (LWFA) can generate electron beams with hundreds of $\mathrm{MeV}$ over only a few millimeters [1-3]. Therefore, they have been considered for many applications, including compact colliders (using staged LWFA) [4], as well as compact synchrotron sources and free-electron lasers [5-8]. Yet, the feasibility of these two applications partly relies on the ability to focus the electron beam in the device. For instance, in the context of multi-stage LWFA, if the electron beam is not matched to the focusing fields in each stage, its emittance will quickly deteriorate [9]. Since the beam diverges at the end of each stage, it should be refocused before entering the next stage. Similarly, when combining a LWFA and an undulator to produce synchrotron radiation, it is important to collimate the electron bunch in order to obtain an intense photon flux [5]. This is even more crucial for a free-electron laser (FEL) [6], for which a low divergence and high charge density is critical to the FEL process itself.

In this context, it was suggested that the bunch could be collimated by an adiabatic matching section, at the end of the accelerator $[10,11]$. However, this solution may be difficult to implement as it requires a long, well-controlled density gradient at the accelerator end, and could be very sensitive to laser depletion and diffraction [10].

Alternatively, the electrons can be focused by quadrupole lenses. Yet, because of their relatively low field gradients, quadrupole lenses typically have to be placed several decimeters behind the accelerator [5]. This raises

*remi.lehe@ensta.fr

cedric.thaury@ensta-paristech.fr

Published by the American Physical Society under the terms of the Creative Commons Attribution 3.0 License. Further distribution of this work must maintain attribution to the author(s) and the published article's title, journal citation, and DOI. several issues for laser-accelerated electron bunches. For example, due to the relatively high divergence and energy spread of these bunches, the duration and normalized emittance can strongly increase in the drift length [12-14]. Moreover, in the context of a multi-stage LWFA, the inter-stage transport lines would be considerably longer than the accelerating stages themselves [4], thereby leading to a substantial overall length for the collider. A compact focusing device is thus highly desirable.

A plasma lens [15], which could typically be placed only millimeters behind a LWFA, could be this compact alternative. In a plasma lens, the bunch travels into a gas jet, where it can ionize and radially expel the gas electronsthereby surrounding itself with a focusing ion cavity. This effect was studied theoretically [15-18] and experimentally [19-24], in the context of conventional accelerators. However, in a plasma lens, there is always a finite length at the bunch head over which the focusing is very nonuniform [18,21,24] (this effect typically gives rise to head erosion). This is a major drawback for the short LWFA bunches, because this finite length can be comparable to the length of the bunch itself [25].

In this article, we propose an alternative focusing scheme, the laser-plasma lens, which is compact but avoids the drawbacks of plasma lenses. It can be used to refocus the beam (e.g., in a multistage accelerator) or to collimate it (e.g., before sending it into a wiggler or into quadrupole lenses). For definitiveness, we will concentrate here on the collimating case, but a refocusing configuration can be obtained by changing the lens parameters.

\section{SINGLE-PULSE LASER-PLASMA LENS}

The idea of the proposed laser-plasma lens is to place a low-density gas jet after the one in which laser-wakefield acceleration took place. The two jets are separated by a millimeter-scale drift space, in which both the laser and the 
electron bunch naturally diverge. When entering the second gas jet, the laser drives a wakefield, in which the trailing electron bunch can be focused and collimated.

First, we notice that, because the laser diverges in the drift space (the typical Rayleigh length is $Z_{\mathrm{R}} \sim 200 \mu \mathrm{m}$ ), $a_{0}$ is low in the second jet, and the wakefield is close to the linear regime. Considering a Gaussian pulse and neglecting beamloading effects, the equation of transverse motion for an electron in this jet is (see Appendix A for a demonstration):

$$
\frac{d p_{x}}{d t}=-\frac{\eta m_{e} c^{2} a_{0}(z)^{2}}{w(z)^{2}} \sin \left(k_{p} d\right) x \mathrm{e}^{-2\left(x^{2}+y^{2}\right) / w(z)^{2}},
$$

where $z$ is the propagation coordinate, $x$ and $y$ the transverse coordinates, $w(z)$ the laser waist, $d$ the distance between the electron and the center of the laser pulse, $n_{2}$ and $k_{p}=$ $\sqrt{4 \pi r_{e} n_{2}}$ are the density and plasma wave vector of the second jet. By definition, $\eta \equiv \sqrt{2 \pi}\left(k_{p} \sigma_{z}\right) \mathrm{e}^{-k_{p}^{2} \sigma_{z}^{2} / 2}$ with $\sigma_{z}$ the RMS length of the laser pulse. To be collimated, the electron must be in a focusing phase of the wakefield $\left(k_{p} d<\pi\right)$, which imposes an upper bound on the density of the second jet: $n_{2}<\pi /\left(4 r_{e} d^{2}\right)$.

Equation (1) can be integrated under a few approximations. First, we assume that the density is step-like:

$$
n(z)=\left\{\begin{array}{lll}
n_{1} & \text { for } z<0 & \text { (First jet) } \\
0 & \text { for } 0<z<L_{\mathrm{d}} & \text { (Drift space) } \\
n_{2} & \text { for } L_{\mathrm{d}}<z<L_{\mathrm{d}}+L_{2} & \text { (Second jet) }
\end{array}\right.
$$

with $L_{\mathrm{d}}$ the drift length and $L_{2}$ the length of the second jet. Since typically $Z_{R} \ll L_{\mathrm{d}}$, we assume $w(z) \approx w(0) \times z / Z_{R}$ and $a_{0}(z) \approx a_{0}(0) \times Z_{R} / z$ in the second jet. We further neglect the variation of the electrons' $\gamma$ factor in the second jet (due to the low $a_{0}$ in this jet), and assume that $x(z), y(z) \ll w(z)$. Equation (1) then leads to

$$
\frac{d^{2} x}{d z^{2}}=-\frac{k_{\mathrm{foc}}^{2} Z_{R}^{2}}{z^{4}} x \quad \text { with } \quad k_{\mathrm{foc}}^{2}=\frac{\eta a_{0}(0)^{2}}{\gamma w(0)^{2}} \sin \left(k_{p} d\right) .
$$

Let us consider an electron that exits the first jet with a transverse position $x_{0}$ and propagation angle $\theta_{0}$, and freely propagates in the drift space. By integrating Eq. (3), its propagation angle $\theta_{x}=d x / d z$ is found to be

$$
\begin{aligned}
\theta_{x}= & \chi \theta_{0}\left[\cos \left(\frac{k_{\mathrm{foc}} Z_{R}^{2}}{L_{\mathrm{d}}}-\frac{k_{\mathrm{foc}} Z_{R}^{2}}{z}+\varphi\right)\right. \\
& \left.-\frac{k_{\mathrm{foc}} Z_{R}^{2}}{z} \sin \left(\frac{k_{\mathrm{foc}} Z_{R}^{2}}{L_{\mathrm{d}}}-\frac{k_{\mathrm{foc}} Z_{R}^{2}}{z}+\varphi\right)\right]
\end{aligned}
$$

where $\varphi=\arg \left[1+x_{0} /\left(\theta_{0} L_{\mathrm{d}}\right)+i x_{0} /\left(k_{\mathrm{foc}} Z_{R}^{2} \theta_{0}\right)\right]$ and $\chi=$ $\left|1+x_{0} /\left(\theta_{0} L_{\mathrm{d}}\right)+i x_{0} /\left(k_{\mathrm{foc}} Z_{R}^{2} \theta_{0}\right)\right|$. This expression is represented in Fig. 1 for arbitrary values of $x_{0}$ and $\theta_{0}$.

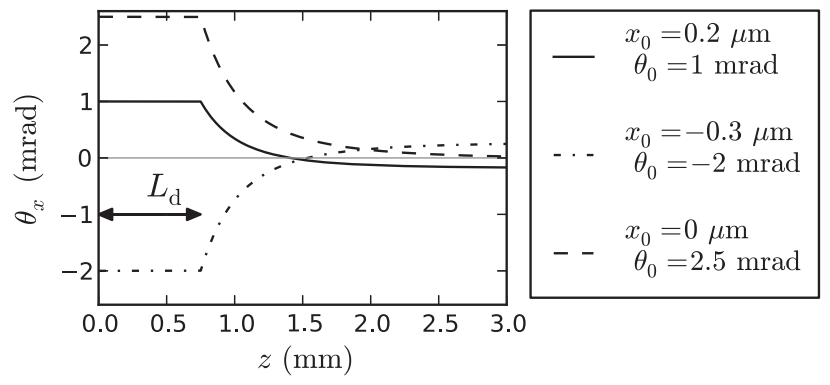

FIG. 1. Evolution of the propagation angle of individual electrons, as predicted by Eq. (4) for several $\left(x_{0}, \theta_{0}\right)$, and for $\gamma=400, Z_{R}=180 \mu \mathrm{m}, a_{0}(0)=5, w(0)=6.7 \mu \mathrm{m}$, and $\eta=1$. In the drift space $\left(z<L_{d}\right)$, Eq. (4) does not apply, and instead $\theta_{x}$ remains constant.

Ideally, for collimation, the second jet should end exactly at a $z$ for which the electron exits with a zero angle i.e., $\theta_{x}\left(L_{\mathrm{d}}+L_{2}\right)=0$. According to Eq. (4), this happens when $\frac{k_{\mathrm{foc}} Z_{R}^{2}}{L_{\mathrm{d}}+L_{2}} \tan \left(\frac{k_{\mathrm{foc}} Z_{R}^{2}}{L_{\mathrm{d}}}-\frac{k_{\mathrm{foc}} Z_{R}^{2}}{L_{\mathrm{d}}+L_{2}}+\varphi\right)=1$. However, in practice, this relation cannot be satisfied simultaneously by all electrons, since $k_{\text {foc }}$ depends on $\gamma$ and since $\varphi$ depends on $x_{0}$ and $\theta_{0}$. Yet, in a typical LWFA, $\varphi \ll 1$ for most electrons and a good compromise is

$$
\frac{\left\langle k_{\mathrm{foc}}\right\rangle Z_{R}^{2}}{L_{\mathrm{d}}+L_{2}} \tan \left(\frac{\left\langle k_{\mathrm{foc}}\right\rangle Z_{R}^{2}}{L_{\mathrm{d}}}-\frac{\left\langle k_{\mathrm{foc}}\right\rangle Z_{R}^{2}}{L_{\mathrm{d}}+L_{2}}\right)=1,
$$

where the brackets denote an average over the energy distribution of the bunch. Equation (5) demonstrates that the density $n_{2}$ (which controls the value of $\eta$ and thus of $k_{\mathrm{foc}}$ ) and the length $L_{2}$ of the second jet have to be tuned in relation with the drift length $L_{\mathrm{d}}$, for optimal collimation. Experimentally, these parameters may not be known precisely, but the tuning can still be done by observing the evolution of the final divergence as one of the parameters (e.g., $n_{2}$ ) is varied. This tuning procedure can also compensate the impact of finite density gradients or of a non-Gaussian laser pulse, which were not considered in the above calculations. [Notice that our scheme is still valid for non-Gaussian pulses, provided that these pulses induce a focusing wakefield force proportional to $x$ close to the axis, as is the case in Eq. (3). In practice, this is true for most realistic laser profiles after a few Rayleigh length.]

Obviously, even if Eq. (5) is satisfied and even if the bunch is monoenergetic, it will still have a finite RMS divergence due to its finite emittance. Assuming that the emittance $\epsilon_{x} \equiv \sqrt{\left\langle x^{2}\right\rangle\left\langle p_{x}^{2}\right\rangle-\left\langle x p_{x}\right\rangle^{2}} / m_{e} c$ is conserved, its value imposes a lower bound on the final divergence: $\sqrt{\left\langle\theta_{x}^{2}\right\rangle} \geq \epsilon_{x} / \gamma \sqrt{\left\langle x^{2}\right\rangle}$ - which is reached when the bunch is optimally collimated. Incidentally, this last inequality shows that the drift space is key to the collimating scheme, since it allows $\left\langle x^{2}\right\rangle$ to increase and thus reduces the lower bound on $\sqrt{\left\langle\theta_{x}^{2}\right\rangle}$. 
Three other effects can further increase the final RMS divergence. These aberrations induce a varying $k_{\text {foc }}$ across the bunch, which can prevent the electrons from simultaneously reaching $\theta_{x}=0$. The first aberration is caused by the energy spread, and leads to a spread in $k_{\text {foc }}$ through its relation with $\gamma$ in Eq. (3). The second aberration is the high-radius nonlinearity of the focusing fields [which was neglected in Eq. (3) and Eq. (4)]. Due to the factor $\exp \left(-2\left(x^{2}+y^{2}\right) / w^{2}\right)$ in Eq. (1), the electrons that are far from the axis experience a weaker focusing field, and thus have a lower effective $k_{\text {foc }}$. Finally, the third aberration is transverse beamloading. If the bunch has a high charge, it can drive a high-amplitude plasma wakefield within the linear laser wakefield [26,27]. In this case, the tail of the bunch experiences the focusing force of this plasma wakefield in addition to that of the laser wakefield, and thus has a higher effective $k_{\text {foc }}$ than the head of the bunch [25].

Particle-in-cell (PIC) simulations were run to confirm these results. We used the quasicylindrical code CALDER CIRC [28], with a resolution $\Delta z=0.016 \mu \mathrm{m}, \Delta r=$ $0.1 \mu \mathrm{m}, \Delta t=0.96 \Delta z$, two azimuthal modes and 20 macroparticles per cell. An extended-stencil Maxwell solver similar to that of [29] was used, so as to limit the spurious growth of emittance due to numerical Cherenkov. Finally, we used a third-order interpolation in time when calculating the $B$ field for the Boris pusher, so as to ensure proper compensation of the $\boldsymbol{E}$ and $\boldsymbol{v} \times \boldsymbol{B}$ terms in the Lorentz force (see appendix B for more information on this point).

The double-jet density profile used in the simulation is shown in Fig. 2. A $0.7 \mathrm{~J}, 30 \mathrm{fs}$ (FWHM) laser pulse is focused into the first gas jet, and then further self-focuses and compresses to $a_{0}=5, w_{0}=6 \mu \mathrm{m}$, before diverging in the drift space with $Z_{R}=150 \mu \mathrm{m}$. A $70 \mathrm{pC}, 1$ fs (RMS)
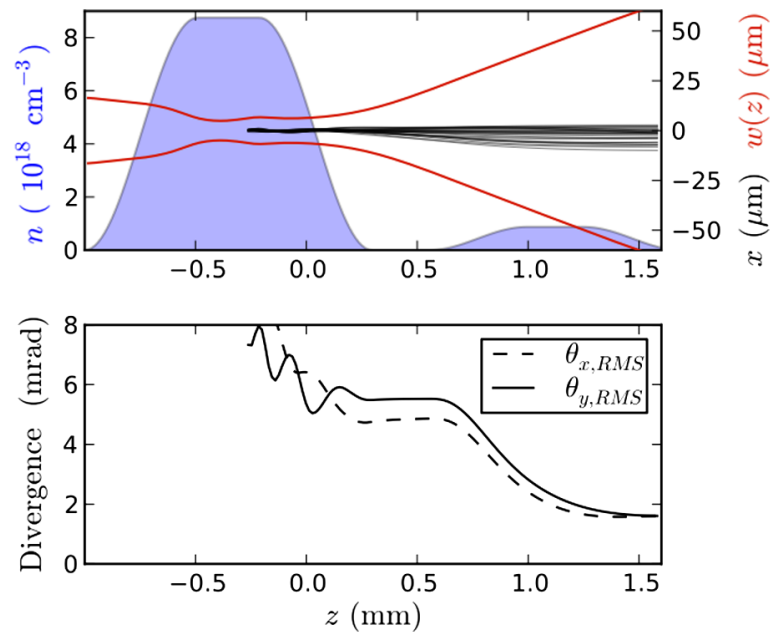

FIG. 2. Upper panel: density profile (blue), laser waist (red), and trajectories of a few injected electrons (black) in the PIC simulation. Lower panel: RMS divergence of the bunch in the $x$ and $y$ directions. (The laser is polarized along $x$.) bunch is self-injected and accelerated up to $\gamma=450$ with a $10 \%$ energy spread. With these values, the criterion (5) for optimal collimation is approximately satisfied. The evolution of the RMS divergence of the bunch is represented in Fig. 2. Its value oscillates in the first jet (due to the betatron oscillations), remains constant in the drift space, and decreases in the second jet. Overall, the laser-plasma lens reduces the divergence by roughly a factor of 3 . Further analysis revealed that the emittance did not grow significantly in the drift space (which is too short for the effects described in [12-14] to be important) but became twice larger after the second jet. This analysis also showed that this increase was due to the above-mentioned beamloading effect. Consistently, an additional simulation featuring a lower charge $(1 \mathrm{pC})$ showed no substantial increase in emittance. Finally, the simulations confirmed that the energy of the bunch was not significantly modified in the second jet.

Although the simulations show that the laser-plasma lens concept is well suited for beams with $\gamma \sim 400$ (i.e., $E \sim 200 \mathrm{MeV}$ ), we notice that it may not be as efficient for much higher energies. This is because the linear wakefield driven by the diffracting laser pulse may be two low to deflect and collimate high-energy beams. In fact, it can be shown that, if $\gamma>0.6 a_{0}(0)^{2} Z_{R}^{4} / L_{\mathrm{d}}^{2} w(0)^{2}$, then no value of $n_{2}$ and $L_{2}$ can satisfy the criterion (5) for optimal collimation (see Appendix $\mathrm{C}$ for a demonstration).

\section{DOUBLE-PULSE LASER-PLASMA LENS}

To avoid this, we propose an alternative setup for the laser-plasma lens, which is better adapted to high energies. The principle is to focus a second laser pulse into the second gas jet, in order to create a fully evacuated bubble. This second pulse copropagates with the first pulse, but precedes it by a few tens of femtoseconds. As can be seen in Fig. 3, the second pulse can drive a bubble, whereas the first pulse alone can only drive a weak, linear wakefield.
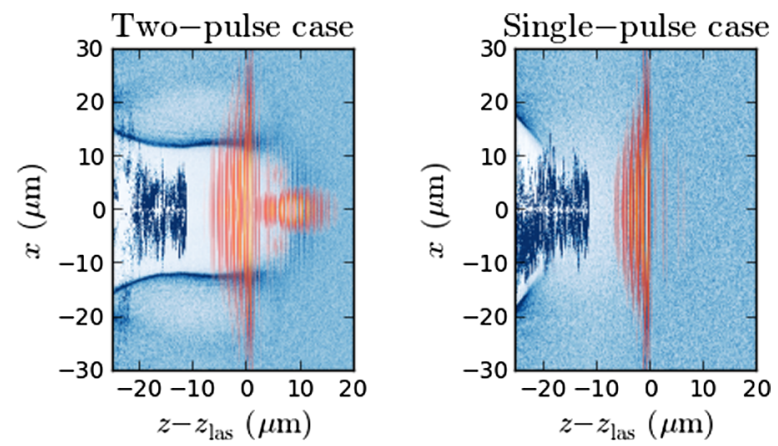

FIG. 3. Aspect of the wakefield in the second jet, during a PIC simulation with (left panel) and without (right panel) a second laser pulse. The electron density and the laser intensity are represented in blue and red respectively. The dark blue spot represents the electron bunch. 
Inside the bubble, the radial force is $F_{r}=-m_{e} c^{2} k_{p}^{2} r / 2$ [30]. This force is much stronger than that of the linear wakefield, and can therefore collimate high-energy electrons. Moreover, the above expression of $F_{r}$ is always linear in $r$ and constant along the bunch-even for high-charge bunches [31]. (This is because the bunch cannot drive its own wakefield inside the fully evacuated bubble, as can be seen in Fig. 3.) Therefore, another advantage is the absence of high-radius and beamloading aberrations with this setup.

With the above expression of $F_{r}$, and the density profile of Eq. (2), the equation of motion in the second jet is $d^{2} x / d z^{2}=$ $-k_{\beta}^{2} x$ where $k_{\beta}=k_{p} / \sqrt{2 \gamma}$ is the betatron wave vector. The corresponding solution for an individual electron is $\theta_{x}=\theta_{0} \cos \left[k_{\beta}\left(z-L_{\mathrm{d}}\right)\right]-k_{\beta}\left(x_{0}+\theta_{0} L_{\mathrm{d}}\right) \sin \left[k_{\beta}\left(z-L_{\mathrm{d}}\right)\right]$. Ideally, the electron should have a zero angle at the end of the second jet, but again, due to the finite energy spread and emittance of the bunch, this relation cannot be satisfied simultaneously by all the electrons. Thus, a reasonable compromise consists in satisfying

$$
\left\langle k_{\beta}\right\rangle L_{\mathrm{d}} \tan \left(\left\langle k_{\beta}\right\rangle L_{2}\right)=1
$$

since, in realistic conditions, $x_{0} \ll \theta_{0} L_{\mathrm{d}}$ for most electrons. With typical parameters $\left(n_{2} \sim 10^{18} \mathrm{~cm}^{-3}, \quad \gamma \sim 10^{3}\right.$, $L_{d} \sim 500 \mu \mathrm{m}$ ), the above relation imposes to use a very thin jet $\left(L_{2} \sim 100 \mu \mathrm{m}\right)$. In practice, such a gas jet is difficult to produce with a standard nozzle, but could be obtained with a free-flowing capillary [32].

To confirm this analysis, we ran a corresponding PIC simulation. The resolution was $\Delta z=0.03 \mu \mathrm{m}, \Delta r=$ $0.2 \mu \mathrm{m}, \Delta t=0.96 \Delta z$, and this simulation was run with a $1.6 \mathrm{~J}$ driving laser pulse and a longer first gas jet, so as to accelerate higher energy electrons. An additional copropagating $1.0 \mathrm{~J}$ laser pulse was focused into the second jet, which had a length $L_{2} \simeq 100 \mu \mathrm{m}$.

Figure 4 displays the density profile along with the waist of each pulse. The first pulse (red solid line) self-focuses in the first jet and triggers self-injection. Conversely, the second pulse (orange dashed line) self-focuses only at the end of the first jet. Thus it reaches the second jet with a low waist, and therefore drives a fully evacuated bubble.

After the first jet, the energy distribution of the electron bunch is wide, and here we consider only its high-energy part (consisting of a $\sim 50 \mathrm{pC}$ peak around $\gamma=900$ with $16 \%$ energy spread). The trajectories of the corresponding electrons and their RMS divergence are shown in Fig. 4. This divergence progressively decreases in the first jet (due to the adiabatic acceleration of the beam [33]), and sharply drops in the second jet. Here again, the second jet reduces the divergence by a rough factor of 3 . In addition, the emittance and the mean energy of the peak were observed to remain roughly unchanged as the bunch went through the second jet.

Notice that the two-pulse laser-plasma lens could in principle also collimate multi-GeV bunches. For a $10 \mathrm{GeV}$
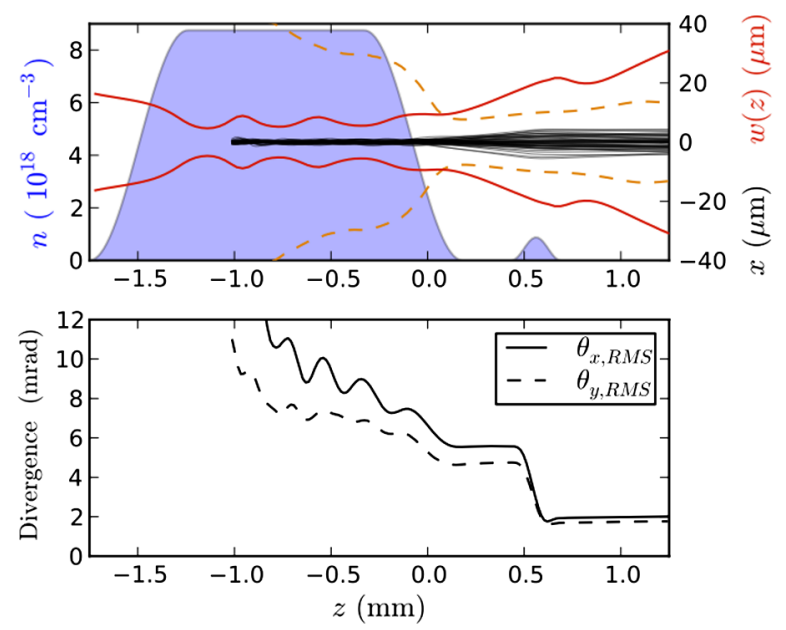

FIG. 4. Upper panel: density profile (blue), waists of the first (red, solid) and second (orange, dashed) laser pulse, and trajectories of a few injected electrons (black) in the PIC simulation. Lower panel: divergence of the bunch.

beam for instance, the criterion (6) is satisfied with, e.g., $n_{2}=3 \times 10^{18} \mathrm{~cm}^{-3}, L_{2}=400 \mu \mathrm{m}$, and $L_{\mathrm{d}}=800 \mu \mathrm{m}-$ which are reasonable, realistic values indeed.

\section{CONCLUSION}

In conclusion, we proposed two experimental schemes, which can both efficiently collimate LWFA electrons. The first scheme uses one laser pulse, is relatively easy to implement experimentally, and is well suited for electrons around $200 \mathrm{MeV}$. The second scheme uses two laser pulses, and can collimate electrons with energies from $\sim 500 \mathrm{MeV}$ to $\sim 10 \mathrm{GeV}$. PIC simulations showed that both schemes can realistically divide the final divergence by 3 ; larger reductions could be possible, using a longer drift length. The obtained low-divergence bunches could then be used directly (e.g., in synchrotron sources) or sent into quadrupole lenses for further collimation. In the latter case, the initial collimation by the laser-plasma lens would drastically reduce the subsequent emittance increase and bunch lengthening that occurs in the drift space of the quadrupole lens [12-14].

\section{ACKNOWLEDGMENTS}

This work was supported by the European Research Council through the X-five project (Contract No. 339128).

\section{APPENDIX A: DERIVATION OF THE EQUATION OF TRANSVERSE MOTION EQ. (1)}

In this appendix, let us rederive the equation of transverse motion for a relativistic electron that experiences the laser-wakefield in the second jet [Eq. (1)]. In its general form, the equation of motion for a relativistic electron propagating along the $z$ axis (i.e., $\boldsymbol{v} \simeq c \boldsymbol{e}_{z}$ ) reads 


$$
\frac{d \boldsymbol{p}}{d t}=-e(\boldsymbol{E}+\boldsymbol{v} \times \boldsymbol{B}) \simeq-e\left(\boldsymbol{E}+c \boldsymbol{e}_{z} \times \boldsymbol{B}\right) .
$$

In our case, the fields $\boldsymbol{E}$ and $\boldsymbol{B}$ are the fields generated in the wake of the laser pulse, as it propagates in the second jet. This pulse is considered here to be Gaussian:

$$
\boldsymbol{a}(\boldsymbol{r}, t)=a_{0} \mathrm{e}^{-\frac{x^{2}+y^{2}}{w^{2}}-\frac{(z-c t)^{2}}{4 \sigma_{z}^{2}}} \cos \left[k_{0}(z-c t)\right] \boldsymbol{e}_{x}
$$

with $a_{0} \ll 1$ (linear regime). In this case, it can be shown $[34,35]$ that the electric and magnetic field in the wakefield are, respectively, of order $a_{0}^{2}$ and $a_{0}^{4}$, and thus that the term $c \boldsymbol{e}_{z} \times \boldsymbol{B}$ is negligible compared to $\boldsymbol{E}$. Moreover, the electric field in the wake of the laser pulse is given by [35]:

$$
\boldsymbol{E}=-\frac{m_{e} c^{2} \omega_{p}}{e} \int_{-\infty}^{t} d t^{\prime} \sin \left[\omega_{p}\left(t-t^{\prime}\right)\right] \frac{\nabla\left\langle\boldsymbol{a}\left(\boldsymbol{r}, t^{\prime}\right)^{2}\right\rangle}{2}
$$

where the brackets denote an average over the fast laser oscillations.

Thus, when projected along the transverse $x$ direction, the equation of motion reads:

$$
\begin{aligned}
\frac{d p_{x}}{d t} & =m_{e} c^{2} \omega_{p} \int_{-\infty}^{t} d t^{\prime} \sin \left[\omega_{p}\left(t-t^{\prime}\right)\right] \frac{\partial_{x}\left\langle\boldsymbol{a}\left(\boldsymbol{r}, t^{\prime}\right)^{2}\right\rangle}{2} \\
& =-\frac{m_{e} c^{2} \omega_{p} a_{0}^{2} x}{w^{2}} \int_{-\infty}^{t} d t^{\prime} \sin \left[\omega_{p}\left(t-t^{\prime}\right)\right] \mathrm{e}^{-\frac{2\left(x^{2}+y^{2}\right)}{w^{2}}-\frac{\left(z-c c^{\prime}\right)^{2}}{2 \sigma_{z}^{2}}} .
\end{aligned}
$$

Carrying out the integration under the assumption that the electron considered is behind the laser pulse (i.e., $\left.c t-z(\equiv d) \gg \sigma_{z}\right)$ leads to

$$
\begin{aligned}
\frac{d p_{x}}{d t} & =-\frac{m_{e} c^{2} \omega_{p} a_{0}^{2} x}{w^{2}} \frac{\sqrt{2 \pi} \sigma_{z}}{c} \sin \left(k_{p} d\right) \mathrm{e}^{-\frac{2\left(x^{2}+y^{2}\right)}{w^{2}}-k_{p}^{2} \sigma_{z}^{2} / 2} \\
& =-\frac{\eta m_{e} c^{2} a_{0}^{2}}{w^{2}} x \mathrm{e}^{-\frac{2\left(x^{2}+y^{2}\right)}{w^{2}}}
\end{aligned}
$$

with $\eta \equiv \sqrt{2 \pi}\left(k_{p} \sigma_{z}\right) \mathrm{e}^{-k_{p}^{2} \sigma_{z}^{2} / 2}$.

\section{APPENDIX B: COMPENSATION OF THE ELECTRIC AND MAGNETIC FORCE TERMS IN THE PIC SIMULATIONS}

In standard PIC simulations of the laser-plasma lens, we observed a strong interaction between the back of the laser pulse and the bunch, which resulted in a progressive growth of divergence throughout the simulation. This effect could have been a major problem for the laser-plasma lens concept if it was indeed physical, but we found that it was in fact of numerical origin. This section describes this artifact, explains its origin, and shows how we corrected it by a better time interpolation of the $\boldsymbol{B}$ field.

\section{Observation of a spurious laser-induced transverse force}

In order to illustrate the above-mentioned interaction between the laser and the electron bunch, let us consider the simulation results of Fig. 5. This figure shows the double-jet density profile (blue curve), along with the evolution of the transverse position (upper panels) and transverse momentum (lower panels) of the accelerated electrons in the $x$ and $y$ directions. Note that, in this simulation, the laser pulse was linearly polarized along the $x$ direction. (This simulation was run with CALDER CIRC and an extended-stencil, Cherenkov-free Maxwell solver, with two azimuthal modes and with $\Delta z=$ $0.03 \mu \mathrm{m}, \Delta r=0.2 \mu \mathrm{m}$ and $c \Delta t=0.96 \Delta z$.) In the drift space, in Fig. 5, the $y$ component of the momenta $\left(p_{y}\right)$ appears to remain roughly constant, but the $x$ component $\left(p_{x}\right)$ varies considerably. As can be seen in the lower left panel, this unexpected variation of $p_{x}$ prevents the electrons from being properly collimated by the second jet, and results in a large final RMS divergence. Thus, this variation represents a major hurdle for the laser-plasma lens concept.

The fact that $p_{x}$ varies while $p_{y}$ remains constant suggests that the observed variations are due to the Lorentz force of the linearly-polarized laser. This is confirmed by Fig. 6, which shows that the electrons are indeed located in a zone where the laser field is relatively important. (The left panel of this figure even shows that the bunch is modulated at the laser wavelength.) In principle, the Lorentz force of the laser pulse can indeed accelerate the electrons transversely and cause their
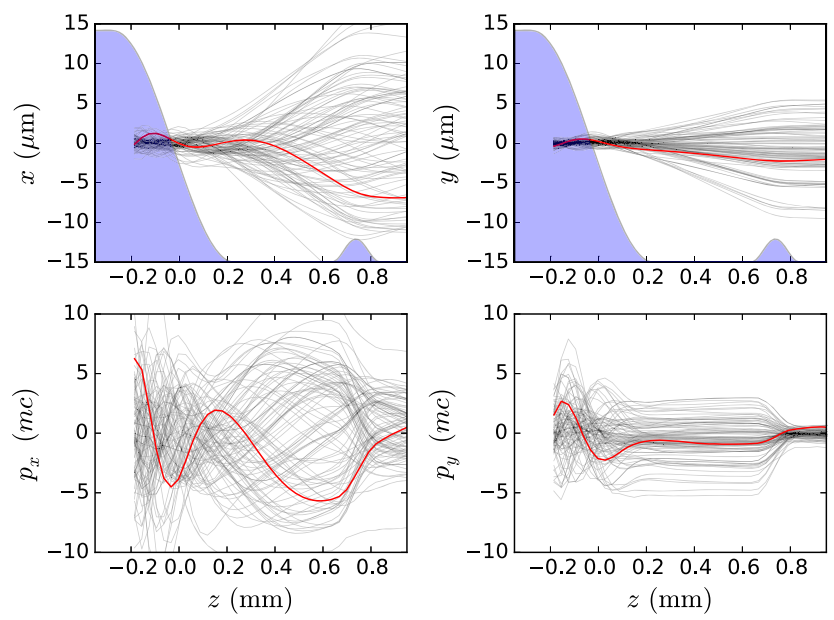

FIG. 5. Results of a standard PIC simulation for the laserplasma lens. Upper panels: Density profile of the two jets (blue curves) and evolution of the transverse coordinates $x$ (left panel) and $y$ (right panel) for some of the accelerated electrons (black lines). (In the simulation the laser is polarized along $x$.) Lower panels: Evolution of the transverse momenta $p_{x}$ (left panel) and $p_{y}$ (right panel) for the same electrons. For more clarity, the trajectory of one of these electrons has been singled out and colored in red. 

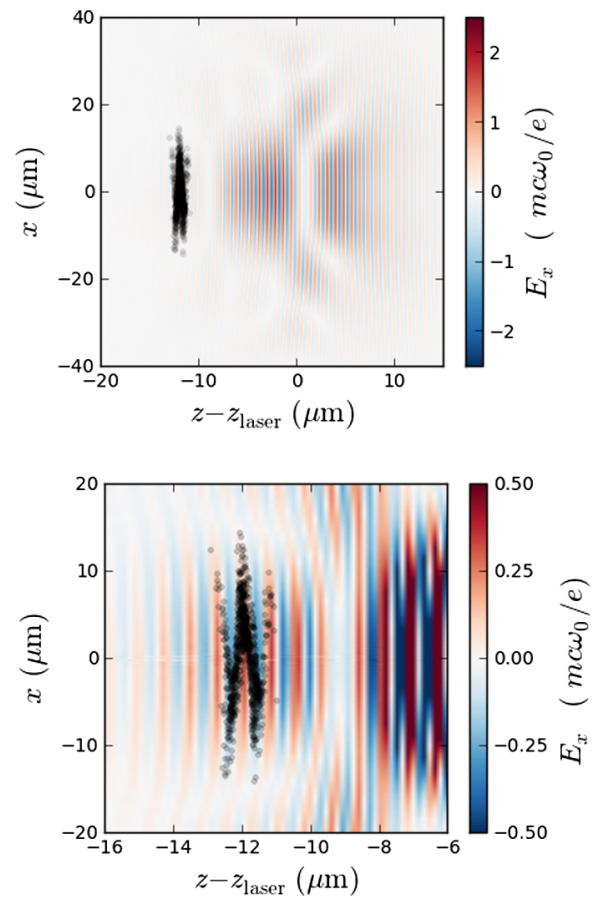

FIG. 6. Top panel: Snapshot of the simulation at $z=550 \mu \mathrm{m}$ (i.e., inside the drift space), showing the laser pulse (red and blue) and the trailing electron bunch (black dots). Bottom panel: Zoom on the electron bunch. (Notice that the electric field $E_{x}$ has also been rescaled.)

momentum to vary along the direction of polarization. However, the amplitude of these variations in the lower left panel of Fig. 6 are strikingly high.

In order to determine whether these variations are of physical or numerical origin, let us estimate the variation of $p_{x}$ that the electrons should physically experience. For an individual electron, the equation of motion in the $x$ direction is (in SI units):

$$
\frac{d p_{x}}{d t}=-e\left(E_{x}-v_{z} B_{y}+v_{y} B_{z}\right) .
$$

Since the laser propagates along the $z$ axis and since the back of the bubble propagates nearly in vacuum (i.e., in the blown-out ion cavity), its fields satisfy $B_{z}=0$ and $B_{y}=E_{x} / c$. This yields:

$$
\frac{d p_{x}}{d t}=-e E_{x}\left(1-\beta_{z}\right) \sim-\frac{e E_{x}}{2 \gamma^{2}}
$$

where $\beta_{z}=v_{z} / c$ and where $1-\beta_{z}$ was evaluated by assuming that the electrons propagate essentially along the $z$ axis. This shows that, for relativistic electrons, the Lorentz force of the laser is proportional to $1 / 2 \gamma^{2}$, which can be very low, since in this simulation $\gamma \sim 300$. Using these estimations, it is possible to evaluate the total physical variation of $p_{x}$ across the drift space. Since the electric field at the position of the electrons is of the order
$\left|E_{x}\right| \sim 0.5 m c \omega_{0} / e$ (see Fig. 6) and since the drift length is of the order $L_{d} \sim 500 \mu \mathrm{m}$, the corresponding variation of $p_{x}$ evaluates as:

$$
\left|\Delta p_{x}\right| \sim \frac{e\left|E_{x}\right|}{2 \gamma^{2}} \frac{L_{d}}{c} \sim 10^{-2} m c .
$$

This value is 500 times lower than the typical variation observed in the simulation: $\left|\Delta p_{x}\right| \sim 5 m c$ (see the lower left panel of Fig. 5). This implies that, because of a numerical artifact, the Lorentz force felt by the electrons in the simulation is unphysically high. Importantly, this unphysical force was observed both with the Cherenkov-free Maxwell solver and the standard Yee solver [36].

\section{A hypothetical origin for the spurious force}

In the above physical calculation, the terms $E_{x}$ and $v_{z} B_{y}$ compensate each other almost entirely $\left(E_{x}-v_{z} B_{y}\right.$ is of the order of $10^{-5} E_{x}$ ). In practice, this implies that $E_{x}$ and $v_{z} B_{y}$ should be calculated with a precision of more than $10^{-5}$ in the simulation, in order to properly capture this compensation. Although this required precision is coarser than machine precision, the intrinsic discretization of the equations in a PIC code can still introduce errors of this order of magnitude.

For instance, Vay [37] showed that, assuming that $\boldsymbol{B}$ is accurately calculated in PIC codes (i.e., in our case that $B_{y}$ equals $E_{x} / c$ with a very good precision), the discretization error on $\boldsymbol{v}$ can lead to an important error on the final force $\boldsymbol{E}+\boldsymbol{v} \times \boldsymbol{B}$. (This error can be corrected for instance by using the Vay pusher [37], instead of the standard Boris pusher [38].) However, in the case of the simulation of Fig. 5, this error was estimated analytically and found to be negligible.

Since the observed spurious force is not due to the discretization error on $\boldsymbol{v}$, it is likely to be due to the calculation of $\boldsymbol{B}$, i.e., to the fact that $B_{y}$ does not equal $E_{x} / c$ with enough precision in the simulation. Here it is important to note that, in the standard Yee algorithm, the relation $B_{y}=E_{x} / c$ is satisfied exactly on the grid [39] for a sinusoidal wave propagating along $z$. However, when computing the Lorentz force $E_{x}-v_{z} B_{y}$ on the particles, the fields $E_{x}$ and $B_{y}$ are interpolated from the grid to the macroparticles. In particular, the magnetic field $B_{y}$ needs to be interpolated in time, since $B_{y}$ is defined at half time steps in the Yee lattice, whereas the Lorentz force $E_{x}-v_{z} B_{y}$ is calculated at integer time steps. In the standard PIC algorithm, this interpolation in time is performed by a simple time average, i.e., the field $B_{y}^{n}$ at integer time step $n \Delta t$ is computed through $B_{y}^{n}=\left(B_{y}^{n+1 / 2}+B_{y}^{n-1 / 2}\right) / 2$. As shown in Fig. 7, this method of interpolation typically introduces an error in the calculation of $B_{y}^{n}$, and thus $B_{y}^{n}$ does not equal $E_{x}^{n} / c$ when interpolated on the macroparticles. 


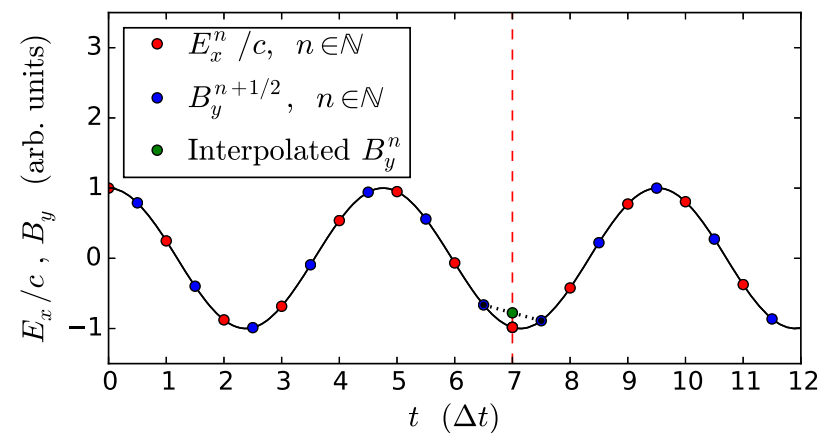

FIG. 7. Schematic evolution of the $E_{x}$ and $B_{y}$ fields for a sinusoidal wave, in a PIC simulation. On the Yee lattice, the discrete fields $E_{x}^{n} / c$ (red dots) and $B_{y}^{n+\frac{1}{2}}$ (blue dots) lie exactly on the same sinusoid. However, when interpolating the $B_{y}$ field to an integer time (dashed red line), the interpolated field $B_{y}^{n}$ is not on the sinusoid (green dot), and thus the $E_{x}$ and $B_{y}$ fields do not cancel exactly when computing the Lorentz force.

This error can be directly evaluated through a Taylor expansion. Assuming that the fields $E_{x}^{n}$ and $B_{y}^{n+1 / 2}$ are of the form $E_{x}^{n}=E_{0} \cos \left[\omega_{0} n \Delta t\right], B_{y}^{n+1 / 2}=$ $\frac{E_{0}}{c} \cos \left[\omega_{0}\left(n+\frac{1}{2}\right) \Delta t\right]$, the interpolated field at integer time step $B_{y}^{n}$ is of the form:

$$
\begin{aligned}
B_{y}^{n} & =\frac{B_{y}^{n+1 / 2}+B_{y}^{n-1 / 2}}{2} \\
& =\frac{E_{0}}{c} \cos \left[\omega_{0} n \Delta t\right] \times\left(1-\frac{\left(\omega_{0} \Delta t\right)^{2}}{8}\right)+O\left(\Delta t^{3}\right)
\end{aligned}
$$

where $\omega_{0}$ is the laser frequency. Thus $B_{y}^{n}$ is only secondorder accurate in $\Delta t$. In the simulation, the corresponding computed force on the electrons is

$$
\begin{aligned}
F_{x}^{n} & =-e\left(E_{x}^{n}-v_{z} B_{y}^{n}\right) \\
& =-e E_{0}\left[\left(1-\beta_{z}\right)-\beta_{z} \frac{\left(\omega_{0} \Delta t\right)^{2}}{8}\right] \cos \left(\omega_{0} n \Delta t\right) .
\end{aligned}
$$

Here the first term is physical, but the second term (which is typically much greater than the first one) is of numerical origin and can explain the spuriously high force along $x$ in Fig. 5.

\section{Confirmation of the origin of the spurious force, and correction by a better interpolation of the $B$ field}

In order to decrease this spurious force, we implemented a third-order accurate interpolation method in our PIC code, instead of the standard second-order accurate method. As shown in Fig. 8, this method uses the values $B_{y}^{n-3 / 2}, B_{y}^{n-1 / 2}$ and $B_{y}^{n+1 / 2}$ in order to compute $B_{y}^{n}$. The exact expression of the interpolated field is

$$
B_{y}^{n}=\frac{3}{8} B_{y}^{n+1 / 2}+\frac{3}{4} B_{y}^{n-1 / 2}-\frac{1}{8} B_{y}^{n-3 / 2}
$$

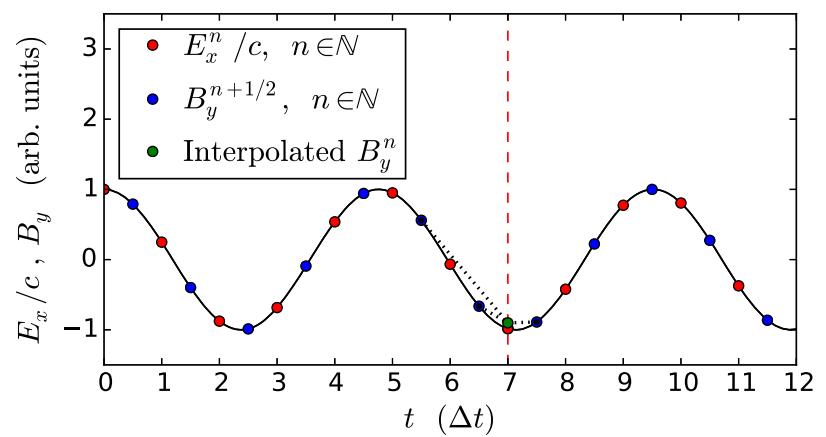

FIG. 8. Schematic representation of the third-order accurate interpolation method. This method is more accurate than the standard second-order accurate interpolation method, and thus the interpolated field lies closer to the sinusoid than in Fig. 7.

where the weighting coefficients of this sum have been chosen so as to have third-order accuracy. By carrying out a Taylor expansion of the above equation (with $B_{y}^{n+m / 2}=$ $\left.\frac{E_{0}}{c} \cos \left[\omega_{0}\left(n+\frac{m}{2}\right) \Delta t\right]\right)$, it can indeed be shown that:

$B_{y}^{n}=\frac{E_{0}}{c} \cos \left[\omega_{0} n \Delta t\right]+\frac{E_{0}}{c} \frac{\left(\omega_{0} \Delta t\right)^{3}}{16} \sin \left[\omega_{0} n \Delta t\right]+O\left(\Delta t^{4}\right)$.

The simulation corresponding to Fig. 5 was rerun with this third-order interpolation method. The results of this simulation and those of Fig. 5 are compared in Fig. 9 and Fig. 10. The bunch still experiences the fields of the back of the laser with the third-order method (see the bottom panel of Fig. 10), but the variations of $p_{x}$ in the drift space are almost negligible (see the lower right panel of Fig. 9). Notice that the proposed third-order interpolation method
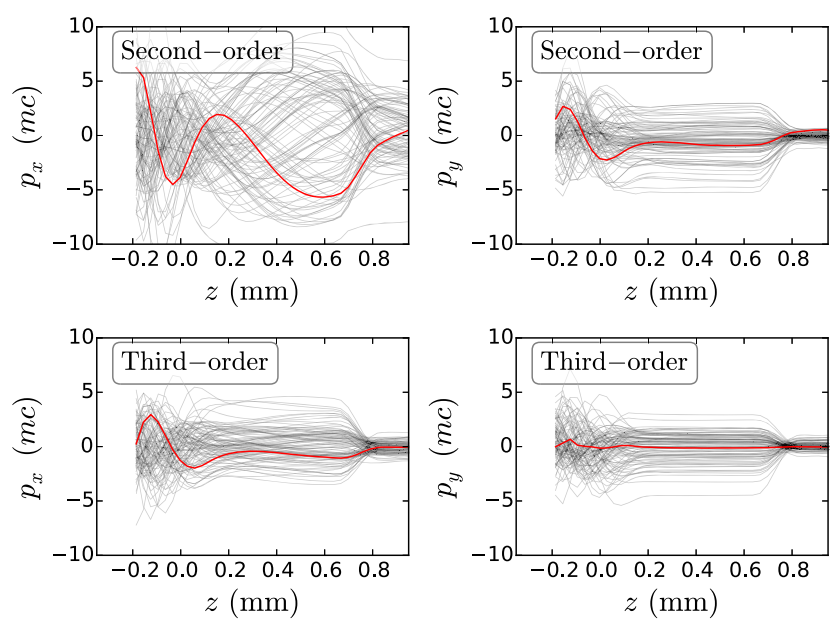

FIG. 9. Comparison of the simulation results with second-order accurate interpolation (top panels) and with third-order accurate interpolation (lower panels). The plots show the evolution of the transverse momenta $p_{x}$ (left panels) and $p_{y}$ (right panels) of the accelerated electrons throughout the simulation. 

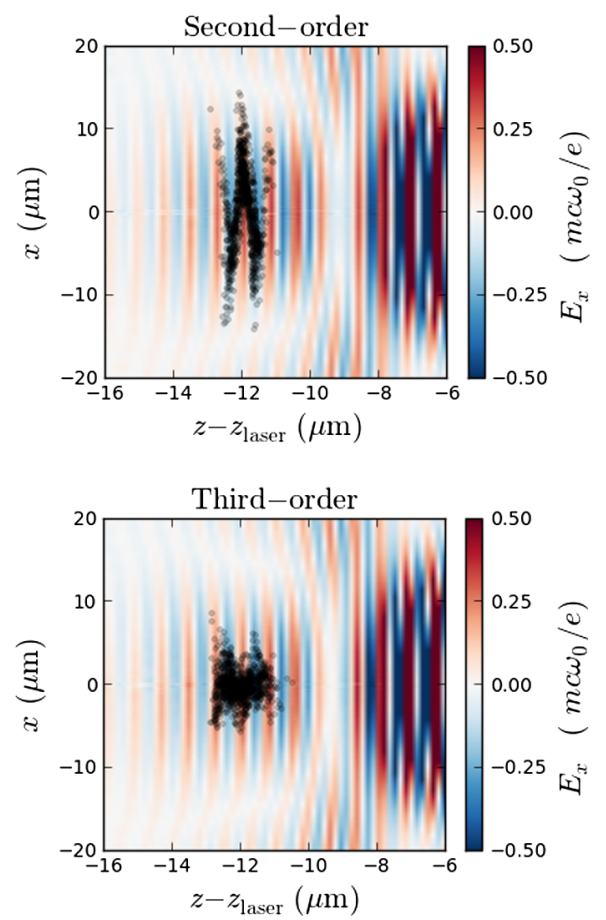

FIG. 10. Snapshot of the simulation at $z=550 \mu \mathrm{m}$ with the second-order accurate (top panel) and third-order accurate (bottom panel) methods. The electric field of the laser is virtually unchanged with the third-order method, but the electrons (black dots) experience a weaker force.

does not attempt to explicitly reduce the Lorentz force of the laser, but only to compute the $B$ field with more precision. Therefore, the results of Fig. 9 confirm that the variations of $p_{x}$ observed in Fig. 5 were unphysical, and that they appeared as a consequence of an inaccurate interpolation of the $B$ field in time, which lead to a lack compensation of the $\boldsymbol{E}$ and $\boldsymbol{v} \times \boldsymbol{B}$ terms in the Lorentz force.

In addition, the lower right panel of Fig. 9 shows that the laser-plasma lens can now properly collimate the electrons in the $x$ direction. This implies that the impact of the laser field on the electron bunch is in reality too weak to represent a serious hurdle for the laser-plasma lens concept. In accordance with this and with the above analysis, the proposed third-order interpolation method was systematically used in the simulations described in the article.

\section{APPENDIX C: DERIVATION OF THE MAXIMAL ENERGY IN THE SINGLE-PULSE SCHEME}

Let us demonstrate here that, if:

$$
\gamma>\frac{4}{\pi} \sqrt{\frac{2}{\pi \mathrm{e}}} \frac{a_{0}(0)^{2} Z_{R}^{4}}{L_{\mathrm{d}}^{2} w(0)^{2}} \approx 0.6 \frac{a_{0}(0)^{2} Z_{R}^{4}}{L_{\mathrm{d}}^{2} w(0)^{2}}
$$

then no value of $n_{2}$ and $L_{2}$ can satisfy the criterion (5) for optimal collimation.
We start by noticing that, whatever the value of $n_{2}$ (and thus of $k_{p}$ ), one has:

$$
\sin \left(k_{p} d\right) \leq 1 \quad \text { and } \quad\left(k_{p} \sigma_{z}\right) \mathrm{e}^{-k_{p}^{2} \sigma_{z}^{2} / 2} \leq \mathrm{e}^{-1 / 2} .
$$

In this case, from its definition in Eq. (3), $k_{\text {foc }}$ satisfies

$$
k_{\mathrm{foc}}^{2}<\sqrt{\frac{2 \pi}{\mathrm{e}}} \frac{a_{0}(0)^{2}}{\gamma w(0)^{2}}
$$

and thus, if $\gamma$ satisfies Eq. (C1), then:

$$
k_{\mathrm{foc}}^{2}<\frac{\pi^{2}}{4} \frac{L_{d}^{2}}{Z_{R}^{4}} \quad \text { i.e. } \quad \frac{k_{\mathrm{foc}} Z_{R}^{2}}{L_{d}}<\frac{\pi}{2} .
$$

Let us now consider the function $f\left(L_{2}\right)=$ $\frac{k_{\mathrm{foc}} Z_{R}^{2}}{L_{d}+L_{2}} \tan \left(\frac{k_{\mathrm{foc}} Z_{R}^{2}}{L_{d}}-\frac{k_{\mathrm{foc}} Z_{R}^{2}}{L_{d}+L_{2}}\right)$, and let us show that its maximum is lower than 1 -i.e., that no value of $L_{2}$ can satisfy the criterion (5) which can be written $f\left(L_{2}\right)=1$.

When $L_{2}$ varies from 0 to $\infty$, the argument of the tangent function varies from 0 to $k_{\mathrm{foc}} Z_{R}^{2} / L_{d}$. Because $k_{\mathrm{foc}} Z_{R}^{2} / L_{d}<$ $\pi / 2$ (as shown above), the function $f$ is continuous, and its maximum is reached when its derivative is zero, i.e., (after some algebra) for $L_{2, \max }$ such that:

$$
\frac{k_{\mathrm{foc}} Z_{R}^{2}}{L_{d}+L_{2, \max }}=\frac{t}{1+t^{2}}
$$

where $t \equiv \tan \left(\frac{k_{\mathrm{foc}} Z_{R}^{2}}{L_{d}}-\frac{k_{\mathrm{foc}} Z_{R}^{2}}{L_{d}+L_{2 \text { max }}}\right)$. Replacing this equation in the expression of $f$, one has

$$
\max (f)=f\left(L_{2, \max }\right)=\frac{t^{2}}{1+t^{2}}<1 .
$$

This thus shows that, if Eq. (C1) is satisfied, no value of $n_{2}$ and $L_{2}$ can satisfy the criterion (5) - since, as mentioned before, this criterion can be written as $f\left(L_{2}\right)=1$.

[1] J. Faure, Y. Glinec, A. Pukhov, S. Kiselev, S. Gordienko, E. Lefebvre, J.-P. Rousseau, F. Burgy, and V. Malka, Nature (London) 431, 541 (2004).

[2] C. G. R. Geddes, C. Toth, J. van Tilborg, E. Esarey, C. B. Schroeder, D. Bruhwiler, C. Nieter, J. Cary, and W. P. Leemans, Nature (London) 431, 538 (2004).

[3] S. P. D. Mangles, C. D. Murphy, Z. Najmudin, A. G. R. Thomas, J. L. Collier, A. E. Dangor, E. J. Divall, P. S. Foster, J. G. Gallacher, C. J. Hooker, D. A. Jaroszynski, A. J. Langley, W. B. Mori, P. A. Norreys, F. S. Tsung, R. Viskup, B. R. Walton, and K. Krushelnick, Nature (London) 431, 535 (2004).

[4] C. B. Schroeder, E. Esarey, C. G. R. Geddes, C. Benedetti, and W. P. Leemans, Phys. Rev. ST Accel. Beams 13, 101301 (2010). 
[5] M. Fuchs, R. Weingartner, A. Popp, Z. Major, S. Becker, J. Osterhoff, I. Cortrie, B. Zeitler, R. Horlein, G. D. Tsakiris, U. Schramm, T. P. Rowlands-Rees, S. M. Hooker, D. Habs, F. Krausz, S. Karsch, and F. Gruner, Nat. Phys. 5, 826 (2009).

[6] K. Nakajima, Nat. Phys. 4, 92 (2008).

[7] H.-P. Schlenvoigt, K. Haupt, A. Debus, F. Budde, O. Jackel, S. Pfotenhauer, H. Schwoerer, E. Rohwer, J. G. Gallacher, E. Brunetti, R. P. Shanks, S. M. Wiggins, and D. A. Jaroszynski, Nat. Phys. 4, 130 (2008).

[8] I. A. Andriyash, R. Lehe, A. Lifschitz, C. Thaury, J. M. Rax, K. Krushelnick, and V. Malka, Nat. Commun. 5, 4736 (2014).

[9] T. Mehrling, J. Grebenyuk, F. S. Tsung, K. Floettmann, and J. Osterhoff, Phys. Rev. ST Accel. Beams 15, 111303 (2012).

[10] C. M. S. Sears, A. Buck, K. Schmid, J. Mikhailova, F. Krausz, and L. Veisz, Phys. Rev. ST Accel. Beams 13, 092803 (2010).

[11] K. Floettmann, Phys. Rev. ST Accel. Beams 17, 054402 (2014).

[12] K. Floettmann, Phys. Rev. ST Accel. Beams 6, 034202 (2003).

[13] A. G. Khachatryan, A. Irman, F. A. van Goor, and K. J. Boller, Phys. Rev. ST Accel. Beams 10, 121301 (2007).

[14] M. Migliorati, A. Bacci, C. Benedetti, E. Chiadroni, M. Ferrario, A. Mostacci, L. Palumbo, A. R. Rossi, L. Serafini, and P. Antici, Phys. Rev. ST Accel. Beams 16, 011302 (2013).

[15] P. Chen, Part. Accel. 20, 171 (1987).

[16] P. Chen, K. Oide, A. M. Sessler, and S. S. Yu, Phys. Rev. Lett. 64, 1231 (1990).

[17] J. J. Su, T. Katsouleas, J. M. Dawson, and R. Fedele, Phys. Rev. A 41, 3321 (1990).

[18] J. B. Rosenzweig, B. Breizman, T. Katsouleas, and J. J. Su, Phys. Rev. A 44, R6189 (1991).

[19] H. Nakanishi, Y. Yoshida, T. Ueda, T. Kozawa, H. Shibata, K. Nakajima, T. Kurihara, N. Yugami, Y. Nishida, T. Kobayashi, A. Enomoto, T. Oogoe, H. Kobayashi, B. S. Newberger, S. Tagawa, K. Miya, and A. Ogata, Phys. Rev. Lett. 66, 1870 (1991).

[20] G. Hairapetian, P. Davis, C. E. Clayton, C. Joshi, S. C. Hartman, C. Pellegrini, and T. Katsouleas, Phys. Rev. Lett. 72, 2403 (1994).

[21] N. Barov, M. E. Conde, W. Gai, and J. B. Rosenzweig, Phys. Rev. Lett. 80, 81 (1998).

[22] R. Govil, W. P. Leemans, E. Y. Backhaus, and J. S. Wurtele, Phys. Rev. Lett. 83, 3202 (1999).

[23] J. S. T. Ng, P. Chen, H. Baldis, P. Bolton, D. Cline, W. Craddock, C. Crawford, F. J. Decker, C. Field, Y. Fukui,
V. Kumar, R. Iverson, F. King, R. E. Kirby, K. Nakajima, R. Noble, A. Ogata, P. Raimondi, D. Walz, and A. W. Weidemann, Phys. Rev. Lett. 87, 244801 (2001).

[24] M. C. Thompson, H. Badakov, J. B. Rosenzweig, G. Travish, N. Barov, P. Piot, R. Fliller, G. M. Kazakevich, J. Santucci, J. Li, and R. Tikhoplav, Phys. Plasmas 17, 073105 (2010).

[25] R. Lehe, C. Thaury, A. Lifschitz, J.-M. Rax, and V. Malka, Phys. Plasmas 21, 043104 (2014).

[26] S. Corde, C. Thaury, K. T. Phuoc, A. Lifschitz, G. Lambert, J. Faure, O. Lundh, E. Benveniste, A. Ben-Ismail, L. Arantchuk, A. Marciniak, A. Stordeur, P. Brijesh, A. Rousse, A. Specka, and V. Malka, Phys. Rev. Lett. 107, 215004 (2011).

[27] K. H. Pae, I. W. Choi, and J. Lee, Phys. Plasmas 17, 123104 (2010).

[28] A. F. Lifschitz, X. Davoine, E. Lefebvre, J. Faure, C. Rechatin, and V. Malka, J. Comp. Physiol. 228, 1803 (2009).

[29] R. Lehe, A. Lifschitz, C. Thaury, V. Malka, and X. Davoine, Phys. Rev. ST Accel. Beams 16, 021301 (2013).

[30] W. Lu, C. Huang, M. Zhou, W. B. Mori, and T. Katsouleas, Phys. Rev. Lett. 96, 165002 (2006).

[31] M. Tzoufras, W. Lu, F. S. Tsung, C. Huang, W. B. Mori, T. Katsouleas, J. Vieira, R. A. Fonseca, and L. O. Silva, Phys. Rev. Lett. 101, 145002 (2008).

[32] Z.-H. He, B. Hou, J. A. Nees, J. H. Easter, J. Faure, K. Krushelnick, and A. G. R. Thomas, New J. Phys. 15, 053016 (2013).

[33] I. Kostyukov, A. Pukhov, and S. Kiselev, Phys. Plasmas 11, 5256 (2004).

[34] L. M. Gorbunov, P. Mora, and T. M. Antonsen, Phys. Plasmas 4, 4358 (1997).

[35] E. Esarey, C. B. Schroeder, and W. P. Leemans, Rev. Mod. Phys. 81, 1229 (2009).

[36] K. Yee, IEEE Trans. Antennas Propag. 14, 302 (1966).

[37] J.-L. Vay, Phys. Plasmas 15, 056701 (2008).

[38] J. Boris, in Proceeding of the Fourth Conference on Numerical Simulations of Plasmas (Naval Research Laboratory, Washington D.C., 1970).

[39] More specifically, it can be shown that the expressions $E_{x_{i+\frac{1}{2}, j, k}}^{n}=E_{0} \cos \left[n \omega_{0} \Delta t-\omega_{0} k \Delta z / c\right]$ and $B_{y_{i, j+\frac{1}{2}, k+\frac{1}{2}}^{n+\frac{1}{2}}}^{n+}=$ $B_{0} \cos \left[\left(n+\frac{1}{2}\right) \omega_{0} \Delta t-\omega_{0}\left(k+\frac{1}{2}\right) \Delta z / c\right]$ on the grid are solution of the discretized Maxwell equations, provided that $B_{0}=E_{0} / c$. (In the notations $E_{x_{i+\frac{1}{2}, j, k}^{n}}^{n}$ and $B_{y_{i, j+\frac{1}{2}, k+\frac{1}{2}}}^{n+\frac{1}{2}}$, the superscript represents the position in time, while the subscripts correspond to the position in $x, y$, and $z$ on the discrete spatial grid.). 